Mens

Revue d'histoire intellectuelle et culturelle

mens

\title{
Rire après les Rébellions : les femmes comme miroir déformant de l'actualité canadienne dans Le Fantasque de Napoléon Aubin
}

\section{Mylène Bédard}

Volume 19, numéro 1-2, automne 2018, printemps 2019

Les années 1840 : rupture ou réarticulation des possibles ?

URI : https://id.erudit.org/iderudit/1070067ar

DOI : https://doi.org/10.7202/1070067ar

Aller au sommaire du numéro

Éditeur(s)

Centre de recherche en civilisation canadienne-française

ISSN

1492-8647 (imprimé)

1927-9299 (numérique)

Découvrir la revue

Citer cet article

Bédard, M. (2018). Rire après les Rébellions : les femmes comme miroir déformant de l'actualité canadienne dans Le Fantasque de Napoléon Aubin. Mens, 19(1-2), 17-39. https://doi.org/10.7202/1070067ar
Résumé de l'article

Comme la presse humoristique s'attribue le mandat d'instruire le peuple en révélant au grand jour une vérité dissimulée, cet article entend examiner la rupture provoquée par l'échec des insurrections par le prisme du journal humoristique Le Fantasque entre 1837 et 1842 . Une attention particulière sera portée à la représentation des femmes, qui sont à la fois l'objet du discours, destinataires et sujets, dans la rhétorique du rire. Celles-ci jouent, en effet, un rôle clé dans l'entreprise de fictionnalisation que mène Napoléon Aubin pour se détourner de l'actualité politique, qui fournit peu de matière à rire sous l'Union. 


\title{
Rire après les Rébellions: les femmes comme miroir déformant de l'actualité canadienne dans Le Fantasque de Napoléon Aubin
}

\author{
Mylène Bédard \\ Université Laval et CRILCQ
}

\section{Résumé}

Comme la presse humoristique s'attribue le mandat d'instruire le peuple en révélant au grand jour une vérité dissimulée, cet article entend examiner la rupture provoquée par l'échec des insurrections par le prisme du journal humoristique Le Fantasque entre 1837 et 1842. Une attention particulière sera portée à la représentation des femmes, qui sont à la fois l'objet du discours, destinataires et sujets, dans la rhétorique du rire. Celles-ci jouent, en effet, un rôle clé dans l'entreprise de fictionnalisation que mène Napoléon Aubin pour se détourner de l'actualité politique, qui fournit peu de matière à rire sous l'Union.

\section{Abstract}

As the humorous press ascribes to reveal in the open a hidden truth in order to educate the people, this article intends to examine the rupture caused by the failure of the insurrections by the prism of the newspaper Le Fantasque between 1837 and 1842. Special attention will be paid to the representation of women, as an object of speech, addressees and subjects in the rhetoric of laughter. They play a key role in the fictionalization of the real in order to turn away from political news that provides little to laugh under the Union. 
Après le choc des Rébellions de 1837-1838, le gouvernement britannique exerce une forte répression sur les patriotes, mais aussi sur les organes de presse. Dans ces circonstances, est-il toujours possible de rire au Bas-Canada ${ }^{1}$ ? Plus encore, pourrait-on, par le rire, cerner la rupture entre les décennies 1830 et 1840 ? Certes, les patriotes n'ont pas fait la révolution en riant, mais les circonstances dramatiques ne seraient-elles pas propices à l'hérö̈sation ou à l'idéalisation du rire, considéré comme l'ultime acte de résistance ou, du moins, comme l'un des derniers remparts de la liberté? Le rire n'est-il pas, comme le souligne Lucie Villeneuve à la suite de Mikhail Bakhtine, "l'âme de la liberté entre les mains du peuple ${ }^{2}$ "? Si c'est le cas, de qui, de quoi peut-on rire? Et comment? Plusieurs terrains sont minés en raison de la censure qui mène de nombreux écrivains et rédacteurs de journaux au cachot de telle sorte que le spectre des cibles potentielles tend à se réduire considérablement ${ }^{3}$. Micheline Cambron remarque d'ailleurs que l'humour, très présent dans les pages du journal Le Canadien dans les premières décennies

1 Interrompu depuis le $1^{\text {er }}$ décembre 1837, Le Fantasque réapparaît en juin 1838 avec cette question du rire: "Comment rire, chers lecteurs; adorables lectrices? Au milieu d'un si triste chaos je n'eusse pu que pleurer; j'aimai mieux gémir en silence, me taire que de vous faire partager ma douleur " (Le Fantasque, 11 juin 1838, p. 79).

2 Lucie Villeneuve, Le "journal-fiction" Le Fantasque de Napoléon Aubin (18371845): formes théatrales et romanesques dans le discours journalistique, thèse de doctorat (études littéraires), Montréal, Université du Québec à Montréal, 2008, p. 172.

3 Une partie importante du contenu provient de journaux étrangers et principalement européens, notamment dans "Les Mélanges». Dans l'édition du $1^{\text {er }}$ octobre 1838 , le rédacteur informe d'ailleurs ses lecteurs à ce sujet: "Nous recevons en ce moment un assez bon nombre des derniers numéros du Figaro de Paris, dont nous tirons les articles qu'on trouvera plus bas. Nous recevrons sous peu, de France, d'autres journaux satiriques et de littérature légère ensorte que nous serons bientôt à même de tenir nos lecteurs au courant de la chronique amusante de Paris et de Londres, à laquelle nous destinerons une petite partie de notre journal et de son feuilleton" (Le Fantasque, $1^{\text {er }}$ octobre 1838, p. 211). L'importation de contenu étranger peut ainsi être conçue comme une stratégie de contournement de la censure. 
du XIX ${ }^{e}$ siècle, se raréfie à partir des événements insurrectionnels 4 . Qu'à cela ne tienne, un rédacteur de journal satirique, Napoléon Aubin, va continuer de fustiger les gouverneurs et les représentants de l'ordre les uns après les autres, mais toujours de manière oblique pour tenter d'échapper à la coupe des censeurs 5 .

Pour cerner la rupture ou la réarticulation des possibles en 1840, je me suis donc penchée sur le cas du Fantasque, journal humoristique fondé pendant l'effervescence des grandes assemblées populaires de l'été 1837 et dont la publication se poursuit jusqu'en 1849. Comme la presse humoristique se pose en véritable porte-parole de la vérité, se donnant pour objectif de dénoncer haut et fort ce que taisent les journaux dits sérieux, Le Fantasque m’est apparu comme un témoin privilégié de la crise politique et, donc, comme un prisme permettant d'observer la brisure provoquée par l'échec des insurrections et l'évanouissement des possibles qu'elles avaient laissé espérer, tant sur le plan politique que littéraire.

Considérant, à l'instar de Guillaume Pinson, que la presse contribue à «structurer les catégories mentales, les perceptions et les pratiques genrées ${ }^{6} »$, je me suis tout particulièrement intéressée à la représentation du féminin dans les pages du Fantasque. J'ai choisi de m'attarder à la place qu'occupent les femmes dans cette feuille

4 Micheline Cambron, "Humour et politique dans la presse québécoise du $\mathrm{XIX}^{\mathrm{e}}$ siècle: des formes journalistiques comme sources d'humour", Bulletin d'histoire politique, vol. XIII, n 2 (hiver 2005), p. 37.

5 À la même époque en France, "[1] a censure, les délits de presse [dont le délit de tendance], les mesures financières [dont le cautionnement] : tout cet attirail répressif explique ou rend possible, à partir des années 1820 et jusqu'en 1868 et 1881 , la constitution d'un espace journalistique "divertissant". C'est au gré de la législation que celui-ci existe et évolue, en contrepoint. [...] Ce paramétrage politique a pour corrélat le travail d'interprétation engagé par le pouvoir en place et le destinataire du quotidien, à l'endroit d'un article de presse toujours potentiellement "duplice" " (Boris Lyon-Caen, "Écrire pour divertir», dans Dominique Kalifa et al. [dir.], La civilisation du journal: histoire culturelle et littéraire de la presse française au XIX siècle, Paris, Nouveau Monde éditions, 2011, p. 795-796).

6 Guillaume Pinson, «La femme masculinisée dans la presse mondaine française de la Belle Époque», CLIO. Histoire, femmes et sociétés, no 30 (2009), p. 211. 
humoristique, qui sont à la fois l'objet du discours, destinataires du journal et sujets ${ }^{7}$. Parce que les femmes avaient su profiter de l'instabilité révolutionnaire de la décennie 1830 pour élargir l'éventail de leurs interventions politiques et culturelles ${ }^{8}$, elles pourraient être, peut-être davantage que les hommes, affectées par le rétablissement et le durcissement de l'ordre social dans les années 1840. L'analyse du traitement médiatique des femmes, à partir du dépouillement des trois premiers volumes du Fantasque, soit d'août 1837 à mars $1842^{9}$, me parait susceptible d'éclairer le repli apparent des femmes dans l'espace domestique après les Rébellions.

\section{Les femmes et la rhétorique duale de Napoléon Aubin}

Le poème publié en première page du numéro inaugural du Fantasque, intitulé "Démocrite" et signé par Napoléon Aubin lui-même, affiche dès le premier vers les intentions du rédacteur: "Rions de tout, c'est mon principe ${ }^{10}$ ». Parmi les cibles évoquées, on retrouve le politique, l'usurier et le savant, mais aussi des figures féminines, comme la "femme qui clabaude», la "vieille fille qui minaude», la prude et «la jeune fillette/Qui vous dit bien innocemment,/(Quoiqu'à sa deuxième amourette;)/“J'ai toujours eu peur

7 Voir Christine Planté et Marie-Ève Thérenty, «Masculin/féminin dans la presse du XIX ${ }^{e}$ siècle: le genre de la critique», dans Muriel Andrin et al. (dir.), Femmes et critique(s): lettres, arts, cinéma, Namur, Presses universitaires de Namur, 2009, p. 15-32.

8 Sur cette question, voir mon ouvrage Écrire en temps d'insurrections: pratiques épistolaires et usages de la presse chez les femmes patriotes (1830-1840), Montréal, Les Presses de l'Université de Montréal, 2016.

9 Je me suis arrêtée en 1842 pour examiner au plus près la transition entre les deux décennies, mais aussi parce qu’à partir du quatrième volume, comme le signale Myriam Côté, «le changement de format du journal», qui «a une influence directe sur l'économie du récit», pourrait entraîner une distorsion dans l'examen des transformations que je m'attachais à repérer (Myriam Côté, Les personnages comme maîtres d'œuvre du récit de l'actualité dans le journal Le Fantasque de Napoléon Aubin [1837-1845], mémoire de maîtrise (littératures de langue française), Montréal, Université de Montréal, 2014, p. 10).

${ }^{10}$ Le Fantasque, $1^{\text {er }}$ août, 1837, p. 1. 
d'un amant ${ }^{11}$ " ". Malgré ce fort contingent féminin parmi les cibles du rire, le poème se termine par ces vers:

Beautés dont j'adore les charmes

Comment hélas! rire de vous

Vos commandements ou vos larmes

Des hommes font autant de fous,

D'ailleurs, je vois un doux sourire.

Toujours accueillir un flatteur;

Ayez donc pitié de la lyre,

Du plus sincère admirateur.

Dans ce poème, on constate, comme le mentionne Lucie Villeneuve, que "[l]'écriture ironique d'Aubin s'appuie sur une parole duale, volontairement ambiguë ${ }^{12}$ ", qui n'épargne pas les femmes. Au contraire, des effets d'échos entre la fiction et l'écriture référentielle contribuent à l'ambiguïté des représentations du féminin selon que le rédacteur se fait séducteur galant ou machiste. Cette parole duale à l'égard des femmes traverse l'ensemble de la première livraison, montrant ainsi que les contradictions et les brouillages, notamment entre réalité et fiction, caractérisent la rhétorique satirique du Fantasque.

Le prospectus, quant à lui, s'attache d'abord à la figure de la lectrice: «Voyez-vous chers lecteurs et aimables lectrices (j'ai la prétention d'avoir des lecteurs et surtout des lectrices) voyez-vous je suis fantasque et c'est tout dire ${ }^{13}$ !» Cette dernière phrase suppose que la volonté d'attirer massivement le lectorat féminin relève de l'extravagance, les journaux de l'époque se préoccupant en général très peu de s'attirer les faveurs du «beau sexe ${ }^{14} »$. Pourtant, la relation

${ }^{11}$ Ibid., p. 1.

${ }^{12}$ Lucie Villeneuve, "Rire et rébellion dans Le Fantasque de Napoléon Aubin (1837-1845), ou Comment se payer la tête à "lord du rhum" ", Bulletin d'histoire politique, vol. XIII, n 2 (hiver 2005), p. 52.

${ }^{13}$ Le Fantasque, $1^{\text {er }}$ août 1837, p. 2.

${ }^{14}$ Voir, à cet égard, le mémoire de maîtrise de Christiane Campagna, Le rôle de la presse selon les propriétaires et rédacteurs des journaux montréalais 1830-1880, Montréal, Université du Québec à Montréal, 1998. 
de prédilection entre le rédacteur et ses lectrices sera maintenue par de nombreuses interpellations et des allusions qui témoignent d'un souci particulier à l'égard de ce public, sur lequel je reviendrai plus tard. La composante féminine est ensuite exploitée dans le prospectus pour illustrer de façon exemplaire le caractère fantasque de la publication:

Je m'engage donc à être fantasque c'est-à-dire: qui va d'un côté de l'autre - qui pense un jour d'une manière et le lendemain d'une autre - fantasque... fantasque, une jeune femme quoi! voilà l'explication. Une jeune femme (et surtout une jolie femme) voyez-vous c'est réellement fantasque, elle rit aujourd'hui de ce qui la fait pleurer demain et qui l'eût fachée hier ${ }^{15}$. N'allez cependant pas me prendre pour une jolie femme, mon Dieu! oui-là vous ne voudriez pas m'entendre parler politique et que deviendrai-je? vous saurez que la politique c'est mon délire! en effet la politique oh! la politique voyez-vous la politique élève l'âme et devient par sa haute importance le seul vrai but pour lequel l'homme fut jeté sur la terre ${ }^{16}$.

Ainsi, la jeune femme constitue l'incarnation des humeurs et des opinions changeantes du Fantasque. Mais très vite, l'incompatibilité entre la figure de la jeune fille, surtout si elle est jolie, et la politique vient rompre cette identification. N'est-ce pas là le propre du fantasque et de la jeune fille d'être instables et volages? Comme «l'humour politique d'Aubin repose [...] sur la traversée des frontières discursives entre nouvelle et commentaire, entre récit de fiction et analyse politique ${ }^{17}$ ", tel que le signale Micheline Cambron, la matière du journal n'est par conséquent pas cloisonnée de façon rigide ni même compartimentée en fonction des genres sexués.

${ }^{15}$ Les citations tirées du journal Le Fantasque sont reproduites sans modification ou correction de notre part.

${ }^{16}$ Le Fantasque, $1^{\text {er }}$ août 1837, p. 2.

${ }^{17}$ Micheline Cambron, "Humour et politique dans la presse québécoise du $\mathrm{XIX}^{\mathrm{e}}$ siècle: des formes journalistiques comme sources d'humour ", Bulletin d'histoire politique, vol. XIII, nº 2 (hiver 2005), p. 39. 
Malgré l'antagonisme qui semble les opposer, femmes et politique seront donc à l'occasion de nouveau mises en relation sous la plume d'Aubin. Les différentes rubriques du journal constituent généralement des espaces de mixité sociale et sexuelle, l'effet humoristique étant bien souvent provoqué par le mélange ou la proximité inusitée entre les classes, les sexes et les domaines d'activité.

Dans ses travaux récents, Marilyn Randall se penche sur la représentation des femmes dans la presse entre 1836 et 1838 et recense de nombreux articles dans lesquels les femmes sont l'objet de procédés de fictionnalisation. Les représentations médiatiques des manifestations patriotiques ou antipatriotiques féminines offrent, en effet, plusieurs exemples d'exagération et de contradiction selon les allégeances des organes de presse. L'effet humoristique dans les "histoires de rire » est souvent créé par un brouillage ou un renversement des genres sexués: "[F]emme masculine ou homme féminin, dans les deux cas, savoir qui porte le pantalon ou la jupe marque habituellement le point culminant de l'histoire. [...] ce qui permet de souligner de façon non équivoque - ou plutôt grâce à l'équivoque - l'équivalence entre "porter le pantalon" [pour une femme] et s'immiscer de façon non appropriée dans l'espace public réservé aux hommes ${ }^{18}$ ". Randall conclut que "la thématique du monde socio-sexuel à l'envers est fréquemment mise au service de la satire politique $^{19}$ ». On trouve effectivement des traces de ce renversement des rôles sexués et de son association à l'actualité politique dans Le Fantasque.

Dans l'édition du $1^{\text {er }}$ novembre 1837, par exemple, un article intitulé «Homme-femme versus femme-homme», qui traite du

${ }^{18}$ Marilyn Randall, «Pantalon, parole et pistolet: l'invention de la femme dans les journaux canadiens à l'époque de la rébellion de 1837-38», dans Marc André Bernier (dir.), Archive et poétique de l'invention, Québec, Éditions Nota bene, 2003, p.163. Voir aussi Marilyn Randall, Les fermes dans l'espace rebelle: histoire et fiction autour des rébellions de 1837-1838, Montréal, Éditions Nota bene, 2013, p. 72-78.

${ }^{19}$ Randall, «Pantalon, parole et pistolet», p. 163. 
cas d'Hortense Globensky-Prévost, une femme ayant manifesté publiquement son obédience politique à la cause des bureaucrates, devient la risée des «braves des Deux-Montagnes ». Pour se défendre de ses assaillants, Globensky-Prévost sort un pistolet de sa robe et devient ainsi l'héroïne des bureaucrates qui lui offrent une théière pour sa bravoure. Le rédacteur conclut que, si les patriotes avaient eu le pouvoir de condamner le geste de la dame, «ils eussent sans doute ordonné à la pauvrette de boire du Whisky tout pur et de porter les habits de son accusateur en même tems qu'ils eussent donné une leçon de courage à celui-ci en le condamnant à se vêtir des jupons de la virile beauté ${ }^{20}$ ». Cette nouvelle qui, malgré son humour, trouve son ancrage dans le régime référentiel fait écho aux Mélanges, publiés en première page, mettant en scène un personnage féminin qui incarne «l'émeute personnifiée, la révolution domestique en permanence» pour convaincre ses parents de consentir à son mariage, au grand désarroi du fiancé qui, comme "martyr et victime", "avait une position dans le monde", mais comme "époux vulgaire» se plaint de n'être plus rien. Ce renversement des identités sexuées où la femme est associée à la révolution et l'homme à l'anonymat cadre tout à fait avec l'esprit de la petite presse humoristique puisque celle-ci, pour reprendre le propos de Jean-Didier Wagneur, se veut le reflet déformant de la réalité en représentant ainsi un monde à l'envers ${ }^{21}$. Le pari de la petite presse avec ces jeux d'inversion consiste néanmoins à révéler une vérité afin d'instruire le peuple. Par le recours à la fiction, il s'agit, dans ce cas-ci, de montrer que la révolution ne produit pas toujours les résultats escomptés. L'association du jupon et du courage tend, par ailleurs, à illustrer les faibles ressources dont disposent les patriotes, qui, sans ce dessous féminin, paraissent complètement démunis.

${ }^{20}$ Le Fantasque, $1^{\text {er }}$ septembre 1837, n ${ }^{\circ} 12$, p. 2.

${ }^{21}$ Jean-Didier Wagneur, «Le journalisme au microscope: digressions bibliographiques", Études françaises, vol. 44, n 3 (2008), p. 23-44. 
Dans l'édition du 10 novembre 1838, au lendemain de la bataille d'Odelltown qui met un terme à la seconde insurrection, on peut lire:

Si l'on en croit nos journaux alarmistes il existe sur les frontières, dans les campagnes et même au sein de nos villes des sociétés secrètes dont les membres sont liés par serment. Quant à moi je ne voudrais pas, pour tout l'or du monde, en faire partie, car une fois embarqué dans ces sociétés mystérieuses votre existence ne tient plus qu'à un fil - le fil de la langue! et il y a tant d'hommes qui sont femmes ${ }^{22}$ !

Le rapprochement entre les femmes et le bavardage et la perfidie sert ici à critiquer les hommes qui dénoncent les patriotes aux autorités. Ainsi, ce sont les traits de caractère associés au féminin qui fournissent des munitions pour se moquer des hommes, des acteurs de l'actualité politique qui plus est. Bien que l'insulte par la féminisation ne soit pas une caractéristique propre aux journaux humoristiques, elle revêt dans ce contexte une dimension autoréflexive. À l'image de la petite presse satirique, le féminin agit comme miroir déformant du masculin, ce qui pourrait expliquer la présence relativement importante des femmes dans Le Fantasque et son association à l'actualité politique.

\section{La figure de la lectrice}

L'effet miroir du féminin se manifeste également par le truchement de la figure de la lectrice. Si la feuille satirique s'identifie à la jeune fille, comme en témoigne le prospectus, la lectrice, elle, vient accentuer les difficultés du métier de journaliste et sert, en quelque sorte, de miroir grossissant à la profession par l'exacerbation de ses problèmes spécifiques. La représentation de la lectrice qu'offre le journal d'Aubin est celle d'un public particulièrement exigeant, prompt à réagir par l'envoi de nombreuses missives, qu'on

${ }^{22}$ Le Fantasque, 10 novembre 1838, p. 259. Voir aussi l'édition du 3 décembre 1838, p. 283. 
peut penser fictives ${ }^{23}$, à chaque faux pas de la rédaction ${ }^{24}$. À l'occasion, des femmes trop bavardes empêchent, en outre, le rédacteur de placer un $\operatorname{mot}^{25}$ et de faire son travail convenablement. En d'autres circonstances, Le Fantasque, menacé par la censure ${ }^{26}$, peine à trouver suffisamment de matière pour couvrir les huit pages de sa livraison. L'absence de liberté de la presse force dès lors le rédacteur, qui se représente régulièrement sous les traits du flâneur, à se tourner vers d'autres sujets, dénichés lors de ses déambulations urbaines ${ }^{27}$. Et comme le rédacteur croise des femmes dans la rue, celles-ci deviennent alors un objet de représentation médiatique jouissant d'une attention soutenue, ce qui n'est pas habituel dans les journaux

${ }^{23}$ Dans les études qu'elles ont consacrées au Fantasque, Myriam Côté et Lucie Villeneuve insistent sur l'usage récurrent que fait Aubin de la fiction épistolaire pour «créer un discours polyphonique qui reproduit le mode de constitution de l'opinion publique» (Villeneuve, Le "journal-fiction" Le Fantasque de Napoléon Aubin [1837-1845], p. 111).

${ }^{24}$ Ce n'est toutefois pas le propre de la lectrice puisque, comme le note Myriam Côté, "plusieurs lecteurs de types différents mentionnent leurs doléances quant au contenu du Fantasque" (Côté, Les personnages comme maîtres d'œuvre du récit de l'actualité, p. 76).

${ }^{25}$ C'est le cas, entre autres, dans la livraison du $1^{\text {er }}$ septembre 1838 , où le rédacteur anticipe la réaction de ses lectrices après avoir confié qu'il avait flâné dans la boutique de Mr. P. Delcour, marchand de cigares: «- Quoi! vous fumez? vont s'écrier toutes mes charmantes lectrices, fi! quelle horreur! quelle peste; oh! le Fantasque sent déjà la pipe, le cigarre! c'est affreux, abominable! peut-être même chique-t-il... Holà! Holà! un moment, mesdemoiselles, ayez la complaisance de brider un instant vos impatientes petites langues et de me donner le tems de placer un mot.» Puis, quelques lignes plus loin, le rédacteur riposte encore devant les récriminations fictives de son lectorat féminin: "Holà! holà! holà encore une fois mes chères lectrices, modérez un instant l'agilité de vos petits moulins à paroles; je vous dirai alors que Mr. Delcour ne vend point son esprit mais qu'il le donne gratis et à tout venant» (p. 178).

${ }^{26}$ Voir, à ce sujet, Jean-Paul Tremblay, À la recherche de Napoléon Aubin, Québec, Presses de l'Université Laval, 1969, p. 41-44.

${ }^{27}$ Au-delà des contraintes liées à la répression politique qui ont une incidence directe sur la matière textuelle du journal, Myriam Côté note que la posture du flâneur "comme source de la nouvelle implique une certaine incertitude quant au contenu du journal, en proie aux désirs du promeneur" (Côté, Les personnages comme maîtres d'auvre du récit de l'actualité, p. 26). 
de l'époque. Or à la censure politique exercée par les autorités britanniques s'ajoute, pour Le Fantasque, la censure des lectrices, ces dernières étant aussi capricieuses que susceptibles, comme on peut le constater dans l'extrait suivant:

Que diable voulez-vous donc que je vous chante, maintenant que tous les sujets me sont ravis ou défendus? si je parle du gouvernement, de la police, des volontaires, des employés publics on me honnit; si j'en dis du mal on m'empoigne pour exciter, dit-on, le peuple à la révolte; si j'en dis du bien, on m'empoigne encore pour exciter à la rebellion en jetant du ridicule sur l'administration [...]; si par hasard j'allais parler du beau sexe, un murmure général de désapprobation s'élèverait contre moi, les dames m'accableraient sans doute de coups de langues et les messieurs de coups de lance. Enfin je ne vois autour de moi que persécution, qu'aridité, que tristesse, qu'incarcérations, que pleurs, que regrets, que grincements de dents et cependant l'on a le cour de me dire, de l'air du monde le plus sérieux, le plus dur: vous ne riez pas assez, riez, dansez, sautez, amusez-nous ${ }^{28}$.

Dans ce texte, la répression des femmes est mise sur un pied d'égalité avec celle du gouvernement qui nuit au traitement des affaires publiques. Le discours sur les femmes semble ici, comme dans d'autres livraisons du Fantasque, fournir de la matière pour remplir les pages du journal. À défaut de pouvoir aborder librement les enjeux de la situation politique canadienne, le rédacteur se replie sur les femmes, et ce, même si c'est pour affirmer qu'il ne peut en parler sans s'attirer les foudres de ces dernières. D'une part, elles n'ont pas le pouvoir de le jeter en prison et, de l'autre, elles servent de prétexte pour éviter de discourir sur les événements dont on ne peut ni ne veut parler directement, soit ceux entourant l'Acte d'Union. Dans l'édition du 12 octobre 1840, après avoir dressé un inventaire de plusieurs pages des superstitions de vieilles dames, le rédacteur se repent:

${ }^{28}$ Le Fantasque, 17 novembre 1838, p. 262. 
Vous me pardonnerez sans doute, indulgents lecteurs, de vous avoir ennuyés des milles balivernes dont je viens de vous entretenir; je l'ai fait pour retracer les milles superstitions qu'on entretient encore dans quelques unes de nos campagnes éloignées, et surtout pour vous écarter un instant de la politique actuelle, qui pour n'en être pas moins folle ni moins extravagante, se trouve infiniment plus ennuyeuse ${ }^{29}$.

Les délais entre la recommandation de lord Durham d'unir le Haut et le Bas-Canada en 1839, l'entérinement de cette loi par le Parlement britannique en juillet 1840 et la mise en application officielle de l'Acte d'Union en février 1841 placent les Canadiens dans l'attente et dans l'incertitude d'un événement à venir à tel point que l'actualité en paraît suspendue. Autrement dit, l'Union ne fournissant pas de matière à rire, mieux vaut explorer d'autres sujets plus légers et, dans ce domaine, les femmes apparaissent comme une source intarissable. Le discours sur le féminin constitue, par ailleurs, un expédient commode pour tenir un métadiscours sur le journal et les conditions de sa fabrication.

Les réactions vives du lectorat féminin décrites par le rédacteur contrastent toutefois avec les qualificatifs qui accompagnent les évocations de la lectrice. Alors que les lecteurs sont désignés par les syntagmes "chers lecteurs" ou "amis lecteurs", les lectrices, elles, sont qualifiées d' «aimables", de "vertueuses", d' «espiègles» et de «sympathisantes». Aux lectrices qui s'offusquent de la description du féminin offerte dans les pages du journal, le rédacteur répond:

Eh! mes demoiselles, calmez-vous. Je ne savais pas seulement que vous fussiez au monde. Si vous entrez dans le cadre de mes descriptions c'est votre faute, non point la mienne; aussi vous montreriez beaucoup plus de bon sens, d'esprit et de jugement si vous étiez les premières à rire ou même à vous moquer de ces folles niaiseries plutôt que de vous fâcher tout rouge comme vous le faites ${ }^{30}$.

\footnotetext{
${ }^{29}$ Le Fantasque, 12 octobre 1840, p. 344.

${ }^{30}$ Le Fantasque, 3 août 1839, p. 52.
} 
Tout porte à croire que les faits et gestes des lectrices sont le fruit d'une certaine affabulation permettant, entre autres, à Aubin de prouver les importants tirages qu'il fait valoir ${ }^{31}$. Le fait que les réactions des lectrices ne soient jamais données à lire tend à conforter l'hypothèse de la fictionnalisation de cette figure, qui sert à la fois de faire-valoir professionnel et de matière de remplissage en période de «disette de nouvelles et de paragraphes ${ }^{32}$ ». Dans plusieurs livraisons, le discours sur les femmes tend, en effet, à empiéter sur l'espace réservé aux événements de l'actualité politique immédiate. Le traitement expéditif en fin de livraison, et en bas de page qui plus est, du sort réservé aux patriotes accusés de haute trahison et de l'Union des Canadas contraste d'ailleurs avec le caractère léger et frivole des

${ }^{31}$ Aubin affirme avoir 1000 abonnés en 1840. Bien que Le Fantasque soit un périodique important durant cette période et qu'il connaisse un succès populaire considérable, on peut qualifier cette affirmation d'hyperbolique considérant que le journal La Minerve a un tirage de 1200 à 1500 copies entre 1832 et 1837 . Le texte intitulé "Fait remarquable» confirme, en outre, la propension d'Aubin à offrir une représentation fictionnalisée de ses lecteurs pour rehausser la qualité de son journal et faire de la réclame: «En jetant l'autre jour un coup-d'œil philosophique et scrutateur sur la liste de nos abonnés des deux sexes, nous avons pu voir avec orgueil que nos lecteurs sont tous des hommes accomplis, des gentilshommes, en un mot la fleur de la population, et que nos lectrices sont exclusivement les dames et demoiselles les plus belles, les plus jolies, les plus aimables de toute la ville, ensorte que nous en sommes venu à la conclusion qu'il n'est pas que des laides, ignorantes et maussades gens qui ne prennent point notre journal, à fort peu d'exceptions près. Il est donc nécessaire que ce qu'il reste d'hommes aimables et de jolies femmes non encore abonnés au Fantasque y souscrivent immédiatement, sans cela ils se trouveront classés avec l'horrible minorité. Afin de ne créer aucun mélange, nous annonçons que nous ne recevrons pas, sous aucun prétexte, au nombre de nos souscripteurs, ceux qui seront dépourvus des précieuses qualités qui distinguent déjà nos lectrices et lecteurs actuels" (Le Fantasque, 27 octobre 1838, p. 244). Alors que dans la livraison du 3 août 1839 , il fera preuve d'exagération en évoquant les réactions nombreuses de son lectorat féminin : «- “Oh! je vous en prie, ne parlez plus de politique!” Voilà ce que me disaient mille suppliantes lectrices à chaque fois que le Fantasque se livrait à d'innocentes railleries aux dépens de quelques uns de nos importants personnages qui se mêlaient de vouloir jouer un rôle impolitiquement politique» (p. 51).

${ }^{32}$ Le Fantasque, 19 octobre 1840, p. 349. 
articles situés dans les premières pages, confirmant le renversement de la hiérarchie des nouvelles et la difficulté à aborder ces sujets traumatisants. C'est le cas, notamment, dans l'édition du 26 décembre 1838. Au bas de la dernière page, sous le titre "Execution", Aubin, généralement très loquace malgré ses lamentations sur la pénurie de nouvelles, écrit: "Cardinal et Duquette ont été exécutés a Montréal vendredi matin 21 Décembre 1838 à 9 heures sur sentence de la cour martiale.» Un espace blanc suit cette nouvelle rapportée dans un style quasi télégraphique que le rédacteur justifie ainsi: «(Je laisse l'espace en blanc afin que mes lecteurs y puissent écrire ce qu'ils croiront convenable; quant à moi je n'en dis rien...... mais je n'en pense pas plus $\left.{ }^{33}\right) »$. Bien que le procédé d'inversion soit caractéristique de la rhétorique humoristique d'Aubin, il n'est vraisemblablement pas utilisé ici pour créer un effet comique. L'espace laissé vacant trahit l'émotion du rédacteur ${ }^{34}$. Les femmes servent donc, dans ce cas-ci, de voie d'évitement en ce qu'elles retardent le traitement des événements sur lesquels le rédacteur a très peu de prise tout en leur laissant peu de place.

\section{Le spectre de la reine}

Parmi les autres voies de contournement de la censure déployées dans Le Fantasque, il y a également la fiction utopique, dont le feuilleton "Mon voyage à la lune », signé par Napoléon Aubin en 1839, constitue un exemple patent. Comme dans son rapport à l'actualité, Aubin prend des libertés avec les codes de l'utopie, entre autres, par la présence d'une protagoniste féminine au premier plan. Si la Lune

${ }^{33}$ Le Fantasque, 26 décembre 1838, p. 108.

${ }^{34}$ Cet extrait n'est pas sans rappeler le clown triste qu'évoque Maxime Prévost, dans son ouvrage Rictus romantiques: «Le sort du clown triste est doublement tragique pour cette raison que, comme tout subalterne, il doit obéir aux volontés d'autrui et - pour comble - il est préposé à la gaieté, au rire, à la bonne humeur. Son métier récuse son destin. Sa profession l'empêche de manifester ses émotions réelles" (Maxime Prévost, Rictus romantiques: politiques du rire chez Victor Hugo, Montréal, Les Presses de l'Université de Montréal, 2002, p. 131). 
est d'abord présentée comme un lieu idéal, le narrateur-voyageur en vient peu à peu à découvrir les injustices qui divisent la population, notamment entre les riches et les pauvres de sorte que les différences entre la Terre et la Lune tendent à s'amenuiser. Toujours dans la perspective d'examiner l'effet miroir créé par le féminin, c'est la figure de la guide du narrateur-voyageur, nommée Bavardine, qui est décrite comme le double du narrateur en raison de son érudition et de son mordant, qui retient mon attention. Le narrateur-voyageur, qui n'est nul autre qu'Aubin lui-même, en vient à envisager la possibilité d'une rédaction bicéphale de son Fantasque:

Cette chère demoiselle parlait comme un beau livre aussi je regrettais beaucoup en l'écoutant de ne pouvoir l'amener avec moi sur la terre pour maider à rédiger mon journal. [...] Quoique la moquerie soit une des principales dispositions du sexe et son occupation favorite, Bavardine excellait tout ce que j'ai vu en ce genre ${ }^{35}$.

Mais l'étroite collaboration qui se dessinait entre la guide et le narrateur-voyageur n'aura pas de suite puisqu'elle semble entravée par le spectre de la reine Victoria, figure qui apparaît à quelques reprises dans les trois premiers volumes du journal et qui se profile à l'occasion derrière les représentations du féminin. En effet, quelques pages plus loin dans la même livraison, on apprend le mariage prochain de la reine d'Angleterre: "Il n'y a qu'une seule chose qui me chagrine là dedans c'est de voir que cette femme va donner le mauvais exemple à toutes ses sujettes, car il n'y a pas de doute qu'elle portera les culottes ${ }^{36}$.» La féminisation du terme «sujet", qui se reflète dans la multitude de figures féminines dépréciées qui parsèment les différentes rubriques du journal, constitue une illustration de la menace de renversement de l'ordre, grammatical et politique, que représente l'association entre le pouvoir et les femmes incarnée par la reine. La possibilité d'une prise de parole féminine

\footnotetext{
${ }^{35}$ Le Fantasque, 17 septembre 1839, p. 76.

${ }^{36}$ Ibid., p. 79.
} 
autonome dans Le Fantasque, d'une union entre la plume masculine et la plume féminine, se trouve ainsi mise en échec par l'image de la reine, figure suprême du féminin et de l'autorité coloniale despotique, qui empêche toute alliance. La description des injustices observées sur la lune agit, en outre, comme une critique voilée du pouvoir colonial britannique personnifié par la reine. À l'utopie s'ajoute donc le détour par le féminin, dont la reine constitue une figure emblématique, dans le traitement oblique de l'actualité politique canadienne. L'insistance avec laquelle le journal représente les femmes sous leur mauvais jour, par leur association à des valeurs négatives, dont la censure, donne à penser que les différents profils féminins dépeints constituent une allégorie du pouvoir impérial, qui annihile la volonté populaire d'autodétermination et entrave la liberté de la presse.

\section{L'apparition du bas-bleu}

À l'instar de la reine, une autre figure féminine représentée comme une source de perturbation apparaît au tournant des années 1840, celle du bas-bleu. En mars, un lecteur du Fantasque, qui signe "Albumophobe», dénonce la nouvelle «manie» des demoiselles canadiennes, celle de tenir un album, qui s'apparente à une rage et fait «le tourment et le désespoir des jeunes gens de Québec en général et de [lui] en particulier ${ }^{37}$ ». La dénonciation de cette pratique féminine importée d'Europe sert non seulement à tourner en dérision les prétentions littéraires des Canadiennes, lesquelles ne maîtrisent pas les règles élémentaires de la sociabilité en quémandant à des inconnus des morceaux en vers ou en prose pour enrichir leur album, mais aussi à valoriser la publication de l'Album du Fantasque ${ }^{38}$, la dénonciation agissant en somme comme

${ }^{37}$ Le Fantasque, 30 mars 1840, p. 120.

${ }^{38}$ L'albumophobe termine sa missive par cette doléance: «La présente, monsieur l'Editeur, est pour prier de vouloir bien m'accepter comme souscripteur à une douzaine de copies de votre album. Par ce moyen je pourrai, à raison de quinze 
artifice publicitaire pour le compte du journal lui-même. Puis, en 1841, un texte tiré de la presse européenne, paru dans les Mélanges, dénonce le ridicule d'un "illustre bas-bleu» qui écrit ses récits de voyage «en courant la poste ${ }^{39}$ ». Cette apparition de la femme de lettres, intellectuelle qui est souvent virilisée en raison de son intérêt pour les champs de compétences masculines (le savoir, l'érudition, les choses de l'esprit, l'écriture publique), m’amène à examiner le dernier pôle de la triade objet, destinataire, sujet. Bien que le rédacteur du Fantasque rapporte souvent des paroles de femmes telles qu'il les a entendues lors de ses flâneries, il ne les donne que rarement à lire. Myriam Côté abonde également dans ce sens lorsqu'elle souligne: "La femme n'est pas celle à qui l'on donne la parole pour exprimer son désir de recevoir un album. Le flâneur fantasque passe plutôt par le désespoir d'un homme devant cet intérêt grandissant des demoiselles pour les albums ${ }^{40}$.» La publication de textes féminins dans les pages du journal est d'ailleurs soumise à des règles spécifiques:

Celles qui ont cru devoir nous adresser leurs plaintes par écrit n'obtiendront de nous aucune justice; car c'est bien le moins que ces inestimables beautés (je suppose naturellement que toutes sont éblouissantes) viennent en personne solliciter la grâce qu'elles nous demandent de vouloir bien livrer à la publicité leurs intéressantes et spirituelles réclamations. Cette règle que nous imposons au meilleur et au plus aimable des deux sexes nous est trop favorable pour que nous nous décidions à nous en départir. Avis donc aux charmants auteurs féminins ${ }^{41}$.

Plutôt que de faire paraître les textes de lectrices dans son journal, Aubin préfère relater le dialogue - lequel constitue un indice de

sous, délivrer ma pauvre tête d'un pénible travail [celui de rédiger des morceaux pour des albums appartenant à des femmes inconnues] sans passer pour un paresseux ou un impoli» (Le Fantasque, 30 mars 1840, p. 120).

${ }^{39}$ Le Fantasque, 15 mars 1841, p. 178.

${ }^{40}$ Côté, Les personnages comme maîtres d'œuvre du récit de l'actualité, p. 76-77.

${ }^{41}$ Le Fantasque, 2 décembre 1840, p. 22-23. 
fictionnalisation - qu'il a eu avec elles afin de servir «l'autofiction journalistique» qu'il met en place, déployant son autoreprésentation "dans un imaginaire autoscopique à fonction réflexive autant que publicitaire ${ }^{42}$ ». Ce procédé intervient également dans les conversations fictives que met en scène Aubin, dont celle entre " $[\mathrm{s}] \mathrm{a}$ bavarde voisine, les naïfs joueurs de dames, la bonne mère de famille et surtout, ô lecteurs!, la bonne, l'intéressante Julie ${ }^{43}$ ", publiée à la suite de la proclamation d'amnistie pour les patriotes exilés. Or l'orchestration de ces différentes voix exclut pourtant celle de Julie: "Je vous dirai de plus que la charmante Julie y mêla sa douce voix, ses spirituelles paroles, mais, en vrai jaloux, je garde tout pour moi. Elle trouva aussi moyen, je ne sais comment de parler du Fantasque et d'en faire un éloge que la modestie m'oblige, quoiqu'à regret, de retrancher ${ }^{44}$.» Le narrateur met en sourdine la parole du personnage féminin, pour mieux faire entendre sa propre voix chantant les éloges de son journal.

À défaut de pouvoir parler librement de l'actualité politique canadienne, le journal se replie sur lui-même et sur ses principaux acteurs: rédacteur, imprimeur, apprenti, lecteurs et lectrices ${ }^{45}$. La transcription d'un dialogue avec une abonnée mécontente, dans la livraison du 2 décembre 1840, est précédée de cette mise en scène autoréflexive :

${ }^{42}$ Sarah Mombert, "La fiction», dans Kalifa et al. (dir.), La civilisation du journal, p. 821. Mombert ajoute, en outre, que cette représentation fictive du journal par lui-même par le biais de ses figures emblématiques est favorisée par le contexte de répression qui suit les tentatives de révolution: «Les espoirs démocratiques déçus par le tournant répressif de la monarchie de Juillet s'expriment par une mise en scène de la communication journalistique où la situation idéale est confrontée à la réalité de la pratique» (p. 821-822).

${ }^{43}$ Le Fantasque, 14 juillet 1838, p. 120.

${ }^{44}$ Ibid., p. 121.

45 Dans son mémoire, Myriam Côté remarque, elle aussi, que "Napoléon Aubin accorde une place importante aux divers représentants des hommes de presse" et que leur intégration au "système du journal» constitue autant d'occasions de développer un métadiscours (Côté, Les personnages comme maîtres d'œuvre du récit de l'actualité, p. 55 et 64). Côté ne lie toutefois pas ce métadiscours à la situation politique canadienne et aux contraintes qu'elle fait peser sur la presse. 
Il était dix heures du matin et nous étions gravement assis dans notre lugubre fauteuil éditorial, rêvant tristement et la larme à l'œil, à ce que nous allions mettre dans notre prochain Fantasque pour faire rire aux éclats un public toujours avide et rarement indulgent. Nous avions réfléchi durant déjà près de cinq heures et nous avions trouvé près de deux idées et demie, juste la moitié d'une idée par heure. Cela n'avançait pas beaucoup pour remplir notre béant journal et cependant j'étais fier de moi-même quand je me comparais à certains grands personnages qui portent la tête bien haut, qui ont le gousset bien garni, qui ont l'air d'être plongés dans de profondes méditations et qui pour cela pensent penser $[\ldots]^{46}$.

Bien qu'il y ait quelques signatures féminines dans les trois premiers volumes du Fantasque, celles-ci visent souvent à discréditer l'auteure et son propos. Les textes sont signés "La femme de..." ou encore "Une folle», ce qui rappelle soit leur rôle domestique et leur subordination à l'autorité du mari, soit leur désordre mental et leur déraison ${ }^{47}$. L'apparition du bas-bleu dans Le Fantasque doit aussi être comprise dans la perspective de l'importation de contenus étrangers à laquelle s'adonne largement Aubin pour remplir les pages de son journal tout en évitant la censure. Elle est en outre tout à fait contemporaine de la condamnation des activités intellectuelles des femmes en général et du bas-bleu en particulier qui s'effectue en France dès le début des années 1840 et se poursuit tout au long de la décennie ${ }^{48}$. On peut citer, à titre d'exemple, Physiologie $d u$

${ }^{46}$ Le Fantasque, 2 décembre 1840, p. 23.

${ }^{47}$ Les lettres d'hommes que publie Aubin dans son Fantasque sont, elles aussi, anonymisées dans le but de façonner un «type de l'homme du peuple plutôt que des individus à part entière». Or les signatures masculines que cite Côté sont plus diversifiées et semblent moins connotées négativement que les signatures féminines ou, à tout le moins, elles renvoient à des figures plus émancipées ou autonomes: «Un requin", «Un intéressé», "Un barbare», "Un patient», " $\mathrm{A}$ volonteer». Côté insiste, par ailleurs, sur l'importance de la signature dans Le Fantasque, car celle-ci participe à l'élaboration du sens (Les personnages comme maîtres d'œuvre du récit de l'actualité, p. 65-66).

${ }^{48}$ Christine Planté, La petite sour de Balzac: essai sur la femme auteur, nouvelle édition, Lyon, Presses universitaires de Lyon, 2015, p. 40-41. 
Bas-Bleu de Frédéric Soulié, un ouvrage paru en 1841 à Paris chez Aubert-Lavigne.

Dans Le Fantasque, cette critique du bas-bleu s'accompagne de la mise en scène, dans les Mélanges, de toute une série de personnages féminins menaçants, de la sorcière à la servante fourbe, qui parviennent à se soustraire à la justice, ce qui force le rédacteur à s'exclamer: "Gode save the Queen». Par différents jeux d'échos entre les discours, les femmes de lettres s'insèrent dans cet imaginaire féminin du crime et du désordre et ne paraissent donc plus considérées comme des alliées dans la création d'une littérature nationale, comme c'était le cas dans Le Populaire trois ans plus tôt. En effet, dans une adresse aux dames publiée dans le premier numéro de ce journal, le rédacteur invitait les Canadiennes à prendre la plume dans l'espace public:

C'est sous ces différens rapports que nous ôsons compter sur le puissant appui des dames. Il en est encore un autre sur lequel il nous est peut-être difficile d'insister; mais nous serons assez hardis pour leur en transmettre le vœu: nous savons, à n'en pas douter, qu'il est beaucoup de dames dans la province qui s'occupent à confier au papier leurs pensées fugitives; si nous pouvions être favorisés de quelques uns de ces essais, nous nous estimerions heureux, et le pays pourrait citer avec orgueil des auteurs dignes de rivaliser avec des noms chers aux lettres en Europe ${ }^{49}$.

Au contraire, dans Le Fantasque, la femme qui écrit n'est pas encouragée à publier; elle suscite plutôt la crainte, voire le mépris. En 1838, le texte intitulé «Le mari d'une femme de lettres » et publié dans les Mélanges décrit de manière nette le renversement des rôles sexués que provoquent les activités littéraires des femmes:

Quand une femme tient la plume dans un ménage, l'ordre de la nature est interverti. C'est la réalisation la plus complète de ce dicton populaire parlant d'un ménage où la femme est le chef

${ }^{49}$ Le Populaire, 10 avril 1837, p. 2. 
de la communauté: - C'est la femme qui porte les sous-pieds. (Sous-pieds est ici rhétoriquement, pour ménager la pudeur des anglaises.) Le mari d'une femme qui écrit des romans, des nouvelles, des historiettes pour les enfans, des comédies, des articles au Journal des Coiffeurs, des devises, des dithyrambes, des dictionnaires et autres menus ouvrages de la littérature courante, le mari de cette femme, disons-nous, doit se résigner à un rôle exceptionnel, et faire abstraction complète de son frac et de sa dignité d'homme. Le cabinet de travail est une pièce qui lui est interdite: le bureau et la bibliothèque sont des meubles qui ne sont pas faits pour lui; mais il peut avoir un boudoir si bon lui semble, et on laisse à sa disposition la toilette et le chiffonnier ${ }^{50}$.

Par ses prétentions intellectuelles, la femme de lettres constitue une transgression de l'ordre traditionnel, du partage sexuel des rôles et de l'espace et contribue à la féminisation de la figure masculine.

\section{Conclusion}

L'étude de la représentation du féminin dans Le Fantasque, par le biais de la triade objet du discours, destinataire du journal et sujet, montre que celle-ci évolue, de 1837 à 1842, de la femme comme miroir du Fantasque lui-même et des acteurs masculins de la scène politique jusqu'à la figure du bas-bleu. Cette métamorphose dans le traitement médiatique des femmes paraît directement liée au déplacement des enjeux de l'actualité. La femme, miroir déformant du masculin par son parti pris politique dans l'espace public, qui provoquait le rire par l'effet de renversement des genres sexués qu'elle entraînait, disparaît au profit d'une représentation tout aussi menaçante pour l'ordre établi : celle de la femme de lettres. La femmehomme ne sert plus de ligne de tir pour se moquer des hommes, mais semble constituer un danger bien réel. Cette menace, d'abord incarnée par la reine Victoria, se matérialise dans la figure du basbleu, qui conjugue également virilité et esprit conquérant, comme

${ }^{50}$ Le Fantasque, février 1838, p. 1. 
en témoigne ce passage du Fantasque consacré à la femme de lettres mistress Trollope, voyageuse associée à Sibylle: «L'œil de mistress Trollope est une sorte de daguerréotype dans lequel viennent se refleter et s'incruster instantanément tous les paysages, toutes les villes, tous les habitans, tous les monumens qu'elle aperçoit - et même qu'elle n'aperçoit pas ${ }^{51}$ !» La biographie de mistress Trollope, nom de plume de Frances Milton, évoque à bien des égards le personnage de Bavardine du feuilleton "Mon voyage à la lune» de Napoléon Aubin et, par extension, le double du rédacteur du Fantasque. D'origine britannique, elle est connue aux États-Unis, où elle voyage d'une ville à l'autre, pour sa description satirique des travers des Américains. Elle use du même mordant lorsqu'elle ausculte les mœurs des Parisiens, dans Paris et les Parisiens. Dans son ouvrage sur Les illustres voyageuses (1866), Richard Cortambert la présente en ces termes:

[C]ette amazone littéraire marche armée d'une critique vive, audacieuse, qui lui amasse des ennemis ardents et d'avide lecteurs. N'attendez pas qu'elle vous raconte les péripéties de toilettes et de boudoirs des contrées qu'elle parcourt, ou plutôt qu'elle habite, car, au détail et à la portée de ses observations, on voit qu'elle ne les visite pas en courant: non, son esprit mâle ne s'arrête pas aux superficies. Quand elle arrive dans un pays, elle y plante son drapeau, et va, la plume haute, attaquer et prôner avec énergie, suivant ses sympathies ou ses haines, les mœurs sociales et politiques qui se déroulent à ses yeux ${ }^{52}$.

En politique comme en littérature, la femme est présentée dans le journal comme celle qui tente d'usurper le pouvoir et les compétences des hommes. Alors que les femmes pouvaient être représentées comme des alliées des patriotes, dans la lutte pour l'émancipation nationale à la fin des années 1830 , que ce soit par l'adoption de

${ }^{51}$ Le Fantasque, 15 mars 1841, p. 178.

${ }^{52}$ Richard Cortambert, Les illustres voyageuses, Paris, Librairie française, 1866, p. 84 . 
résolutions patriotiques ou par leur contribution au projet de création d'une littérature nationale, Le Fantasque les présente plutôt comme des opposantes: au travail journalistique (les lectrices), à la démocratie (la reine) et au partage des rôles sexués (le bas-bleu). Malgré l'évolution observée dans la représentation du féminin, les femmes fictives - inventions masculines - continueront d'occuper massivement l'espace médiatique dédié au féminin, laissant ainsi bien peu de place aux lectrices et aux femmes de lettres réelles ${ }^{53}$. La dimension transgressive associée au féminin dans les pages du Fantasque empêche en somme toute alliance entre celles-ci et les hommes. L'effet miroir du féminin peut ainsi être conçu comme une manière détournée de mettre en lumière les périls et la dégradation auxquels s'exposent les Canadiens sous l'Union.

${ }^{53}$ Ce que confirme Julie Roy dans son article publié dans le présent dossier. 